\title{
Rastreamento de violência contra pessoas idosas: associação com estresse percebido e sintomas depressivos em idosos hospitalizados
}

\author{
Violence against elderly people screening: association with perceived stress and depressive symptoms \\ in hospitalized elderly \\ Detección de violencia contra personas mayores: asociación con estrés percibido y síntomas depresivos \\ en ancianos hospitalizados
}

Isabela Granado Antequera ${ }^{1}$ (c) Maria Carolina Barbosa Teixeira Lopes $^{1}$ (D) Ruth Ester Assayag Batista ${ }^{1}$ (1) Cassia Regina Vancini Campanharo ${ }^{1}$ (1) Paula Cristina Pereira da Costa $^{1}$ (i) Meiry Fernanda Pinto Okuno ${ }^{1}$ (b)

1. Universidade Federal de São Paulo, Escola Paulista de Enfermagem. São Paulo, SP, Brasil. Autor correspondente:

Paula Cristina Pereira da Costa

E-mail: paulinhapcosta@hotmail.com

Recebido em 20/05/2020.

Aprovado em 09/09/2020.

DOl:https://doi.org/10.1590/2177-9465-EAN-2020-0167

\section{Resumo}

Objetivo: Avaliar a prevalência e fatores associados à violência em idosos hospitalizados. Método: Estudo transversal realizado com 100 idosos internados. Aplicaram-se os instrumentos: H-S/EAST, Escala de Estresse Percebido e Escala de Depressão Geriátrica. Utilizou-se análise descritiva para a caracterização sociodemográfica e clínica. Para associar a idade ao H-S/EAST foi utilizado o teste de Mann-Whitney; estresse percebido com o H-S/EAST foi utilizado o teste T, e sintomas depressivos com o H-S/EAST foi utilizado o teste da Razão Verossimilhança. Resultados: A média de idade dos idosos foi 70,39 e 56,0\%, houve risco aumentado para violação de direitos pessoais, características de vulnerabilidade e situações potencialmente abusivas. Os fatores associados ao risco aumentado para violação de direitos pessoais ou abuso direto nos idosos foram maior idade, apresentar estresse percebido e ter sintomas de depressão leve a severa. Conclusão e implicação para a prática: Esses resultados mostram, portanto, a importância da observação atenta do idoso por parte do enfermeiro para permitir a identificação do risco para violência ou violação de direitos. O que possibilita estabelecer ações preventivas, coordenadas com a participação dos demais profissionais bem como o encaminhamento correto de cada situação, cumprimento do dever legal da profissão e do papel cidadão.

Palavras-chave: Idoso; Violência; Depressão; Hospitalização; Enfermagem.

\section{Abstract}

Objective: To assess the prevalence and factors associated with violence in hospitalized elderly. Method: Cross-sectional study conducted with 100 hospitalized elderly. The following instruments were applied: H-S / EAST, Perceived Stress Scale and Geriatric Depression Scale. Descriptive analysis was used for the sociodemographic and clinic characteristics. To associate age with H-S / EAST, the Mann-Whitney test was used; perceived stress with H-S / EAST the T test was used; and depressive symptoms with H-S / EAST the Likelihood Ratio test was used. Results: The average age of the elderly was 70.39 and $56.0 \%$, there was an increased risk for violation of personal rights, characteristics of vulnerability and potentially abusive situations. The factors associated with increased risk for violation of personal rights or direct abuse in the elderly were older age, perceived stress and symptoms of mild to severe depression. Conclusion and implication for practice: These results show, therefore, the importance of careful observation of the elderly by the nurse to allow the identification of risk for violence or violation of rights. This allows establishing preventive actions, coordinated with the participation of other professionals as well as the correct referra of each situation, compliance with the legal duty of the profession and the citizen role.

Keywords: Aged; Violence; Depression; Hospitalization; Nursing

\section{REsumen}

Objetivo: Evaluar la prevalencia y los factores asociados a la violencia en los ancianos hospitalizados. Método: estudio transversal con 100 ancianos hospitalizados. Se aplicaron los instrumentos: H-S / EAST, Escala de estrés percibido y Escala de depresión geriátrica. Se utilizó el análisis descriptivo para la caracterización sociodemográfica y clínica. Para asociar la edad con el H-S / EAST, se utilizó la prueba de Mann-Whitney; estrés percibido con el H-S / EAST se utilizó la prueba T; y los síntomas depresivos con el H-S / EAST se utilizó la prueba de relación de probabilidad. Resultados: La edad promedio de los ancianos era de 70,39 y el $56,0 \%$, había un mayor riesgo de violación de los derechos personales, características de vulnerabilidad y situaciones potencialmente abusivas. Los factores asociados con un mayor riesgo de violación de los derechos personales en los ancianos fueron la edad más avanzada, el estrés percibido y los síntomas de depresión de leve a severa. Conclusión e implicación para la práctica: Estos resultados muestran, por lo tanto, la importancia de la observación cuidadosa de los ancianos por parte de la enfermera para permitir la identificación del riesgo de violencia o violación de los derechos. Esto permite establecer acciones preventivas, coordinadas con la participación de los demás profesionales, así como la derivación correcta de cada situación, el cumplimiento del deber legal de la profesión y el rol ciudadano.

Palabras clave: Anciano; Violencia; Depresión; Hospitalización; Enfermería. 


\section{INTRODUÇÃO}

No Brasil, desde 1940, a população idosa tem altas taxas de crescimento populacional, e estima-se que, em 2040, haverá 153 idosos para cada 100 pessoas com menos de 15 anos. ${ }^{1}$ Diferente do que ocorre em muitos países desenvolvidos, o aumento da população idosa brasileira é progressivo e rápido, o que aponta novos desafios para o setor de saúde, devido à mudança do perfil epidemiológico do país. ${ }^{1}$ A carga de limitações físicas e cognitivas decorrentes da senilidade e senescência bem como os conflitos intergeracionais que esses indivíduos possam vivenciar aumentam sua vulnerabilidade às enfermidades sociais, dentre as quais, destaca-se a violência. ${ }^{2}$

O Ministério dos Direitos Humanos divulgou que, em 2018, houve mais de 37 mil denúncias de violações contra a pessoa idosa pelo Disque 100 (Disque Direitos Humanos), como negligência; abuso financeiro e econômico; violência psicológica, física e sexual contra pessoas idosas. ${ }^{3} \mathrm{O}$ estado de São Paulo respondia por $21,59 \%$ dessas denúncias. Estudo realizado em Unidades de Pronto Atendimento do interior da Paraíba identificou uma prevalência de $69,17 \%$ de risco de violência contra os idosos atendidos por meio do instrumento Hawlek-Sengstock Elder Abuse Screening Test (H-S/EAST). ${ }^{4}$

Segundo a Lei no 12.461 de 2011, considera-se violência contra o idoso qualquer ação ou omissão praticada em local público ou privado que lhe cause morte, dano ou sofrimento físico ou psicológico. ${ }^{5}$ Ela pode ser representada na seguinte tipologia: física, psicológica, sexual, abandono, negligência, financeira e autonegligência. ${ }^{5}$

A literatura aponta que o idoso necessita, muitas vezes, de cuidados e de atenção especial de um ou mais membros da família. Com isso, pela fragilidade da situação em que se encontra, deposita-se a confiança no familiar/cuidador, que pode vir a tornar-se o agressor/violador de seus direitos, transgredindo-os através da violação física, psicológica ou patrimonial, dentre outras situações/casos. Ao passo que, por questões culturais, os idosos frequentemente não acreditam que têm os seus direitos violados, já que são vítimas de seus próprios familiares. ${ }^{6}$

A temática de violência contra o idoso ganhou visibilidade, no Brasil, a partir da década de 1990 com a promulgação e regulamentação da Política Nacional do Idoso e, posteriormente, com a aprovação do Estatuto do Idoso e o Plano de Ação de Enfrentamento da Violência contra a Pessoa Idosa, que tornou obrigatória a notificação de suspeita ou confirmação de todas as formas de violência pelos profissionais de saúde. ${ }^{5}$

A literatura aponta que a depressão é altamente prevalente nas pessoas idosas (15\%) e aumenta para $60 \%$ nos idosos institucionalizados. Muitas vezes o transtorno depressivo está associado à violência. ${ }^{7}$ Parte integrante desse cenário é o maior nível de estresse percebido e que esteja ligado aos quadros com maior nível de depressão. ${ }^{8}$ Além disso, esses fatores causam perda da autonomia e agravamento de quadros patológicos preexistentes no idoso, assim como os fatores sociais que interferem na capacidade funcional, do autocuidado e nas suas relações sociais. ${ }^{9}$

No âmbito da saúde, o envelhecimento populacional requer do sistema de saúde uma organização assistencial contínua e multidisciplinar, que renove o processo de trabalho, assegurando a realização de ações e serviços de saúde que, por sua vez, promovam a qualidade de vida desses indivíduos, envolvendo a saúde física e o bem-estar social e psicológico. ${ }^{1,10}$ No entanto, os profissionais de saúde são desprovidos de orientações claras para a detecção e avaliação da violência contra o idoso, pois, até à data, não existe um consenso acerca do rastreio dos fatores de risco para a ocorrência de violência. $O$ que dificulta o reconhecimento de estar perante uma pessoa idosa vítima de violência, uma vez que é comum as próprias vítimas negarem a existência de maus-tratos. ${ }^{11}$

A violência contra os idosos, além de ser uma violação grave dos direitos humanos que requer ação urgente, é um grande problema de saúde pública que resulta em sérias consequências para a saúde das vítimas, incluindo risco aumentado de morbidade, mortalidade, institucionalização e internação hospitalar e tem um efeito negativo nas famílias e na sociedade, em geral. ${ }^{12}$

O fenômeno da violência sugere um campo maior de investigação, dada a vulnerabilidade e os riscos aos quais o idoso é submetido. Os serviços de saúde são espaços privilegiados para detectar a violência devido à sua proximidade com a população e à sua ampla cobertura. Neste sentido, o hospital apresenta um enorme potencial para programar ações de prevenção, detecção precoce e acompanhamento desse idoso em situação de violência, uma vez que suas atividades tendem a estreitar as relações entre o serviço de saúde e a família, facilitar a identificação de idosos em situação de risco, possibilitar o levantamento das possíveis redes sociais de apoio disponíveis e permitir uma prática transdisciplinar satisfatória. ${ }^{13}$

A participação dos profissionais de enfermagem é de extrema importância na identificação de violência e maus-tratos contra os idosos, identificando situações de risco, com base em observação atenta da comunicação e do comportamento desse idoso, o que permitirá reduzir e combater as situações de violência. ${ }^{13}$

Identificar a violência e seus fatores de risco é imprescindível para redução e cessação dos casos, fornecendo à vítima a assistência adequada à saúde e subsidiando políticas públicas para prevenção de violência. Isto é particularmente importante no caso dos profissionais de enfermagem que, no ambiente de hospitalização, têm grande proximidade com o paciente durante todo o período de internação. Dessa forma, o objetivo dessa pesquisa foi avaliar a prevalência e fatores associados à violência em idosos hospitalizados. 


\section{MÉTODO}

Estudo epidemiológico, transversal e analítico, realizado no Hospital São Paulo (HSP) -hospital de ensino da Universidade Federal de São Paulo (Unifesp) - em unidades clínicas e cirúrgicas. O processo utilizado para selecionar os indivíduos incluídos na amostra foi por conveniência, e o número de participantes foi definido pelo tempo em que foi realizada a coleta de dados, no período de junho de 2019 a fevereiro de 2020. Compondo, assim, a amostra final de 100 idosos. No estudo foram incluídos idosos com idade a partir de 60 anos, capazes de compreendere responder aos questionários, sem qualquer registro de demência em prontuário e que concordaram em participar do estudo e assinaram o Termo de Consentimento Livre e Esclarecido. Para a obtenção dos dados, utilizou-se um questionário estruturado com informações sobre idade, sexo, cor da pele, escolaridade, estado civil, ocupação, dias de hospitalização, renda familiar, presença de cuidador e comorbidades.

Foram também utilizadas as Escalas de Hawlek-Sengstock Elder Abuse Screening Test (H-S/EAST) ${ }^{14}$ para avaliar o risco de violência contra o idoso, de Estresse Percebido ${ }^{15}$ e de Depressão Geriátrica. ${ }^{16}$ Todas as escalas são validadas e adaptadas transculturalmente para o idioma brasileiro.

O Hawlek-Sengstock Elder Abuse Screening Test (H-S/ EAST) é um instrumento composto de 15 itens que cobrem três principais domínios do tema violência contra o idoso: violação evidente de direitos pessoais ou abuso direto, características de vulnerabilidade e situações potencialmente abusivas. Avalia a violência instalada ou presumida com base na perspectiva do próprio idoso. Atribui-se um ponto para cada resposta afirmativa, à exceção dos itens 1, 6, 12 e 14, em que o ponto é dado para a resposta negativa. $\mathrm{O}$ escore de três ou mais pontos pode indicar risco aumentado de algum tipo de violência presente. ${ }^{14}$

A Escala de Estresse Percebido que mensura o estresse percebido dos idosos possui 14 questões com opções de resposta que variam de 0 a 4 ( $0=$ nunca, 1 - quase nunca, 2 - às vezes, 3quase sempre, 4-sempre). As questões com conotação positiva $(4,5,6,7,9,10$ e 13) têm sua pontuação somada invertida da seguinte forma $(0=4,1=3,2=2,3=1,4=0)$. As demais são negativas e devem ser somadas diretamente. $O$ total da escala é a soma das pontuações destas 14 questões e os escores podem variar de zero a 56 ; quanto mais próximo de 56 pontos maior é o estresse. ${ }^{15}$

A Escala de Depressão Geriátrica avalia os sintomas depressivos. Na contabilização do escore, atribui-se um ponto para cada resposta afirmativa, à exceção dos itens 1, 5, 7, 11 e 13 , em que o ponto é dado para a resposta negativa, onde seis ou mais respostas indicam presença de sintomas depressivos. $O$ escore varia de 0 a 15 pontos, em que 0 a 5 pontos indica quadro psicológico normal, 6 a 10 pontos indica quadro de depressão leve e 11 a 15 pontos indica quadro de depressão severa. ${ }^{16}$
Diariamente, foi solicitada ao setor de internação a lista de pacientes com idade a partir de 60 anos e internados em unidades clínicas e cirúrgicas do HSP. Em seguida, a pesquisadora dirigiase a cada local, consultava os prontuários para certificar-se da capacidade do pesquisado para entender e responder aos questionários e instrumentos da pesquisa e depois fazia contato para verificar se preenchiam os demais critérios de inclusão. Os idosos confirmados eram convidados a fazer parte do estudo e, quando concordavam, eram entrevistados individualmente. A leitura dos instrumentos foi realizada pela pesquisadora em um único momento, com duração média de 30 minutos.

Utilizou-se análise descritiva para a caracterização sociodemográfica e econômica, além de dias de hospitalização, presença de cuidador e comorbidades. Para associar as variáveis contínuas ao H-S/EAST foi utilizado o teste de Mann-Whitney e para associar as variáveis categóricas ao $\mathrm{H}$-S/EAST utilizou-se o teste Qui-Quadrado; para associar o estresse percebido ao $\mathrm{H}$-S/EAST foi utilizado o teste $\mathrm{T}$ e para associar a ocorrência de sintomas depressivos ao $\mathrm{H}-\mathrm{S} / \mathrm{EAST}$ foi utilizado o teste da Razão de Verossimilhança. Foi utilizado um nível de significância de $5 \%$ ( $p$-valor $<0,05$ ) e o programa utilizado para a análise foi o Statistical Package for the Social Sciences, versão 19.

Este estudo foi aprovado pelo Comitê de Ética em Pesquisa da Unifesp (CAAE: 05900919.1.0000.5505), número do parecer: 3.156.971.

\section{RESULTADOS}

A média de idade dos idosos foi 70,39 anos ( $D P=7,18)$. A maioria era do sexo masculino, de cor branca, casado, com ensino fundamental, aposentado/pensionista, sem cuidador, com média de internação de 12,20 dias, a morbidade mais prevalente foi hipertensão arterial sistêmica e o medicamento mais utilizado foi o anti-hipertensivo (Tabela 1).

$\mathrm{Na}$ Tabela 2, observa-se que $56,0 \%$ dos pesquisados apresentaram risco aumentado para violação de direitos pessoais ou abuso direto, características de vulnerabilidade e situações potencialmente abusivas. Em relação ao estresse percebido 39,0\% apresentaram pontuação acima do valor médio da escala, o que pode indicar presença de estresse. E 44,0\% apresentaram quadro de depressão leve a severa.

$\mathrm{Na}$ Tabela 3 pode-se verificar que os idosos com risco aumentado para violação de direitos pessoais ou abuso direto, características de vulnerabilidade e situações potencialmente abusivas tinham maior idade. As demais variáveis não foram estatisticamente significantes.

Pacientes com risco aumentado para violação de direitos pessoais ou abuso direto, características de vulnerabilidade e situações potencialmente abusivas obtiveram maior pontuação média na escala de estresse percebido e quadro psicológico de depressão leve a severa (Tabela 4). 
Tabela 1 - Características sociodemográficas, econômica e clínicas dos idosos hospitalizados. São Paulo, Brasil, 2019 (n=100)

\begin{tabular}{|c|c|}
\hline Variáveis & n (\%) \\
\hline Idade (anos) média (DP) & $70,39(7,18)$ \\
\hline \multicolumn{2}{|l|}{ Sexo } \\
\hline Masculino & $56,00(56,00 \%)$ \\
\hline Feminino & $44,00(44,00 \%)$ \\
\hline \multicolumn{2}{|l|}{ Cor } \\
\hline Branca & $45,00(45,00 \%)$ \\
\hline Preta & $18,00(18,00 \%)$ \\
\hline Parda & $34,00(34,00 \%)$ \\
\hline Amarela & $3,00(3,00 \%)$ \\
\hline \multicolumn{2}{|l|}{ Estado civil } \\
\hline Casado & $55,00(55,00 \%)$ \\
\hline Solteiro & $15,00(15,00 \%)$ \\
\hline Viúvo & $15,00(15,00 \%)$ \\
\hline Divorciado & $15,00(15,00 \%)$ \\
\hline \multicolumn{2}{|l|}{ Escolaridade } \\
\hline Não letrado & $11,00(11,00 \%)$ \\
\hline $\begin{array}{l}\text { Ensino fundamental } \\
\text { incompleto }\end{array}$ & $15,00(15,00 \%)$ \\
\hline Ensino fundamental & $40,00(40,00 \%)$ \\
\hline Ensino médio & $19,00(19,00 \%)$ \\
\hline Ensino superior & $15,00(15,00 \%)$ \\
\hline \multicolumn{2}{|l|}{ Emprego } \\
\hline Empregado & $18,00(18,00 \%)$ \\
\hline Desempregado & $6,00(6,00 \%)$ \\
\hline Do lar & $9,00(9,00 \%)$ \\
\hline Aposentado & $67,00(67,00 \%)$ \\
\hline $\begin{array}{l}\text { Renda familiar (reais) } \\
\text { (mínimo-máximo) }\end{array}$ & $1.000,00$ a $10.000,00$ \\
\hline \multicolumn{2}{|l|}{ Presença de cuidador } \\
\hline Sim & $40,00(40,00 \%)$ \\
\hline Não & $60,00(60,00 \%)$ \\
\hline $\begin{array}{l}\text { Internação (dias) média } \\
\text { (DP) }\end{array}$ & $12,20(15,90)$ \\
\hline \multicolumn{2}{|l|}{ Morbidades } \\
\hline $\begin{array}{l}\text { Hipertensão Arterial } \\
\text { Sistêmica }\end{array}$ & $77,00(77,00 \%)$ \\
\hline Diabetes Mellitus & $35,00(35,00 \%)$ \\
\hline Neoplasias & $28,00(28,00 \%)$ \\
\hline \multicolumn{2}{|l|}{ Medicamentos } \\
\hline Antihipertensivos & $77,00(77,00 \%)$ \\
\hline Hipoglicemiantes & $33,00(33,00 \%)$ \\
\hline Antiulcerosos & $17,00(17,00 \%)$ \\
\hline
\end{tabular}

\section{DISCUSSÃO}

Neste estudo houve prevalência de homens. Este resultado pode estar relacionado à menor adesão às práticas de autocuidado por parte dos homens, colocando-os diante de maiores chances de serem hospitalizados, uma vez que a literatura aponta que os homens são resistentes em relação ao cuidado da sua saúde por questões ligadas ao gênero como sentimentos de medo $e$ vergonha. ${ }^{17}$

A média de idade de 70,3 anos é consonante com a faixa etária de estudos relacionados com violência sofrida por idosos. ${ }^{9,18}$ Observou-se no presente estudo que idosos com risco aumentado para violação de direitos pessoais ou abuso direto, características de vulnerabilidade e situações potencialmente abusivas tinham maior idade.

Dos idosos entrevistados, a maioria faz uso de medicações contínuas, informação harmônica com a literatura. Uma pesquisa encontrou relação entre medicamentos e depressão, em que quanto maior a relação medicamento e idoso, maior a razão de chance para depressão. ${ }^{8}$ Projetando esse cenário à realidade da presente pesquisa, o risco para violência aumenta ainda mais, uma vez que os sintomas de depressão geram maior vulnerabilidade no idoso, podendo interferir na capacidade funcional, no autocuidado e nas suas relações sociais.

A morbidade mais prevalente neste estudo foi a HAS. A hipertensão arterial é a doença crônica mais comum entre as pessoas idosas, pois as alterações próprias do envelhecimento fazem com que os idosos estejam mais propensos ao seu desenvolvimento. ${ }^{19} \mathrm{Em}$ consonância ainda com outro estudo que mostrou que ter doenças crônicas é um critério preponderante para casos de violência contra a pessoa idosa, na medida em que esta pode gerar um grau de estresse permanente, o que por si só, é um importante fator de risco para as doenças crônicas. ${ }^{20}$

Aposentadoria foi referida pela maioria dos entrevistados e a renda familiar dos pesquisados variou de um a nove e meio salários mínimos. A maior renda pode propiciar melhores cuidados preventivos e menores rendas podem comprometer o acesso do idoso aos serviços de saúde, ${ }^{21}$ contudo, a amplitude de renda vista nessa amostra sugere um cenário heterogêneo e que em ambos, existe risco para violação dos direitos pessoais, podendo ser ele relacionado à violência financeira, ou não.

Quanto à escolaridade, os dados dessa pesquisa identificaram que $40 \%$ tinham ensino fundamental completo, informação consonante com um estudo realizado em Ribeirão Preto, em que a maioria das vítimas possuía o primeiro grau completo, porém a maioria era constituída de mulheres. ${ }^{22}$

O estado conjugal mais prevalente nessa amostra é o casado, dado consonante com uma pesquisa realizada em Minas Gerais, na qual houve predominância de idosos casados vítimas de violência. ${ }^{18}$ No entanto, outras literaturas sugerem que o casamento possa ser um fator positivo para o envelhecimento mais saudável e que a ausência do companheiro também possa ser apontada como fator para negligência. ${ }^{23} \mathrm{Em}$ contrapartida, outro estudo mostrou que o grau de parentesco que mais está 
Tabela 2 - Risco para violação de direitos pessoais ou abuso direto, características de vulnerabilidade e situações potencialmente abusivas, estresse percebido e sintomas de depressão de idosos hospitalizados. São Paulo, Brasil, 2019 ( $n=100$ )

\begin{tabular}{ccc}
\hline Escalas & Pontuação & $\mathrm{n}(\%)$ \\
\hline \multirow{2}{*}{ H-S/EAST* } & De 0 a 2 pontos & $44,0(44,0 \%)$ \\
Escala Estresse & 3 ou mais pontos & $56,0(56,0 \%)$ \\
& De 0 a 28 pontos & $61,0(61,0 \%)$ \\
& De 29 a 56 pontos & $39,0(39,0 \%)$ \\
Escala de Depressão Geriátrica & Quadro psicológico normal & $56,0(56,0 \%)$ \\
& Quadro de depressão leve & $38,0(38,0 \%)$ \\
& Quadro de depressão severa & $6,0(6,0 \%)$ \\
\hline
\end{tabular}

* Hawlek-Sengstock Elder Abuse Screening Test

Tabela 3 - Características sociodemográficas e econômica relacionadas com risco aumentado para violação de direitos pessoais ou abuso direto dos idosos hospitalizados. São Paulo, Brasil, 2019 ( $n=100)$

\begin{tabular}{|c|c|c|c|}
\hline \multirow{2}{*}{ Variáveis } & \multicolumn{2}{|c|}{ Hawlek-Sengstock Elder Abuse Screening Test } & \multirow{2}{*}{ p-valor } \\
\hline & 0 a 2 pontos & 3 ou mais pontos & \\
\hline Idade (anos) média (DP) & $68,34(6,21)$ & $72,00(7,53)$ & $0,0161^{*}$ \\
\hline Renda familiar (reais) média (DP) & $3619,41(2166,17)$ & $3814,89(2190,33)$ & $0,6334^{*}$ \\
\hline \multicolumn{4}{|l|}{ Sexo } \\
\hline Masculino & $26,00(46,40 \%)$ & $30,00(53,60 \%)$ & $0,5810 * *$ \\
\hline Feminino & $18,00(40,90 \%)$ & $26,00(59,10 \%)$ & \\
\hline \multicolumn{4}{|l|}{ Estado civil } \\
\hline Casado & $29,00(52,70 \%)$ & $26,00(47,30 \%)$ & $0,0559 * *$ \\
\hline Solteiro & $3,00(20,00 \%)$ & $12,00(80,00 \%)$ & \\
\hline Viúvo & $4,00(26,70 \%)$ & $11,00(73,30 \%)$ & \\
\hline Divorciado & $8,00(53,30 \%)$ & $7,00(46,70 \%)$ & \\
\hline \multicolumn{4}{|l|}{ Cor de pele } \\
\hline Branca & $19,00(42,20 \%)$ & $26,00(57,80 \%)$ & $0,9543 * *$ \\
\hline Preta & $8,00(44,40 \%)$ & $10,00(55,60 \%)$ & \\
\hline Parda & $16,00(47,10 \%)$ & $18,00(52,90 \%)$ & \\
\hline Amarela & $1,00(33,30 \%)$ & $2,00(66,70 \%)$ & \\
\hline \multicolumn{4}{|l|}{ Presença de cuidador } \\
\hline Sim & $18,00(45,00 \%)$ & $22,00(55,00 \%)$ & $0,8693 * *$ \\
\hline Não & $26,00(43,30 \%)$ & $34,00(56,70 \%)$ & \\
\hline \multicolumn{4}{|l|}{ Escolaridade } \\
\hline Não letrado & $4,00(36,40 \%)$ & $7,00(63,60 \%)$ & $0,7083^{* *}$ \\
\hline Ensino fundamental incompleto & $6,00(40,00 \%)$ & $9,00(60,00 \%)$ & \\
\hline Ensino fundamental & $16,00(40,00 \%)$ & $24,00(60,00 \%)$ & \\
\hline Ensino médio & $11,00(57,90 \%)$ & $8,00(42,10 \%)$ & \\
\hline Ensino superior & 7,00 (46,70\%) & $8,00(53,30 \%)$ & \\
\hline
\end{tabular}

* teste de Mann-Whitney, ${ }^{* *}$ teste Qui-Quadrado 
Tabela 4 - Associação entre o risco para violação de direitos pessoais ou abuso direto, características de vulnerabilidade e situações potencialmente abusivas, estresse percebido e sintomas de depressão de idosos hospitalizados. São Paulo, Brasil, 2019 ( $\mathrm{n}=100$ )

\begin{tabular}{llccc}
\hline \multirow{2}{*}{ Escalas } & & \multicolumn{2}{c}{ Hawlek-Sengstock Elder Abuse Screening Test } & p-valor \\
\cline { 3 - 4 } & & 0 a 2 pontos & 3 ou mais pontos & 30,64 (8,77) \\
\hline Escala de Estresse & Média (Desvio Padrão) & $23,93(8,84)$ & $21,00(37,50 \%)$ & $0,0003^{*} 0,0003^{* *}$ \\
EDG* & Quadro psicológico normal & $35,00(62,50 \%)$ & $30,00(78,90 \%)$ \\
& Quadro de depressão leve & $8,00(21,10 \%)$ & $5,00(83,30 \%)$ \\
\hline
\end{tabular}

EDG- Escala de Depressão Geriátrica, **teste T, ***teste da Razão de Verossimilhança

envolvido nas agressões contra os idosos está relacionado com o dos próprios cônjuges. ${ }^{24}$

No que se refere aos cuidadores, a amostra refletia uma realidade pouco presente, o que pode contribuir para negligência, recusa e omissão de cuidados necessários ao idoso ou abandono, o que sugere características de vulnerabilidade, apontadas em outra literatura. ${ }^{22} \mathrm{~A}$ ausência de cuidados adequados por parte do cuidador também configura violência contra os idosos, além disso, a limitação de apoio do Estado e da sociedade às famílias de idosos dependentes torna-se uma das condições da gênese da violência. ${ }^{2}$

A hospitalização pode ter grande impacto sobre a pessoa idosa, favorecendo declínio funcional por fatores iatrogênicos como excessivo tempo restrito ao leito, privação do sono, isolamento, desnutrição e desenvolvimento de pensamentos negativos. ${ }^{25,26}$ No presente estudo, a média de internação foi longa, esse tempo deve ser visto pelos profissionais da saúde são só como uma oportunidade para restaurar a saúde física do idosos, como também um momento de identificar fatores de risco para abuso direto, além de traçar linhas de cuidado e acompanhamento pós-alta e realizar as notificações necessárias, previstas em lei.

Verificou-se quadro de depressão leve a severa em $44 \%$ dos pacientes. A depressão é uma das doenças crônicas mais prevalentes nessa faixa etária e está associada ao comprometimento da capacidade funcional e qualidade de vida, gastos financeiros, maior procura dos serviços de saúde, além de ter, muitas vezes, o suicídio como desfecho. ${ }^{27} \mathrm{O}$ comprometimento da capacidade funcional pode estar relacionado ao achado neste estudo, no qual idosos que apresentaram maior pontuação para quadro psicológico de depressão leve a severa tinham risco aumentado para violação de direitos pessoais ou abuso direto.

Com relação ao estresse percebido, 39,0\% apresentaram pontuação acima do valor médio da escala, resultado que vem parcialmente ao encontro de outra pesquisa que descreve os estressores vivenciados por idosos hospitalizados. O envelhecimento é marcado pela diminuição das reservas fisiológicas e doenças crônicas e pode tornar os idosos mais suscetíveis ao estresse, relacionando-o à hospitalização, o que amplia ainda mais o sentimento de perda. ${ }^{25}$
Ainda que seja um tema relevante, as publicações sobre violência contra a pessoa idosa no Brasil mostram-se ainda incipientes. Esse fenômeno pode ser relacionado com a dificuldade da abordagem do tema e com a formação de um escudo cultural que impede a aceitação e o reconhecimento dos fatos.

Como limitação do estudo, ressalta-se o fato de a amostra ter sido coletada em um único serviço, o que pode não representar substancialmente outras realidades, todavia, esses resultados podem apoiar a prática, uma vez que o serviço de saúde deve ser visto como um local privilegiado para investigar violação de direitos, já que os fatores explicitados na presente pesquisa têm grande relevância na identificação da violência.

\section{CONCLUSÃO E IMPLICAÇÕES PARA A PRÁTICA}

Os resultados deste estudo contribuem para alertar as instituições de ensino e serviços de saúde sobre a necessidade de instrumentalizar o estudante/enfermeiro para a investigação e identificação de idosos com risco para violação de direitos pessoais ou abuso direto, características de vulnerabilidade e situações potencialmente abusivas. Neste estudo, a maioria dos idosos apresentou risco aumentado para violação de direitos pessoais ou abuso direto, características de vulnerabilidade e situações potencialmente abusivas e presença de estresse. Idosos com maior idade tiveram risco aumentado para violação de direitos pessoais ou abuso direto. $E$, àqueles com risco aumentado para violação de direitos pessoais ou abuso direto obtiveram maior pontuação média na escala de estresse percebido e quadro psicológico de depressão leve a severa.

No tocante a este cenário, o estresse, como consequência da longevidade ou não, deve ser visto como um fator importante para a vulnerabilização do idoso e não deve ser tratado com naturalidade, visto que nesse estudo, pacientes com risco aumentado para violação de direitos pessoais obtiveram maior pontuação média na escala de estresse percebido.

O enfermeiro tem papel fundamental na preservação da integridade da pessoa idosa e na identificação de situações de violência, além de ter maior probabilidade de se deparar com situações de risco para violência contra os idosos por estar no cuidado direto ao paciente de forma quase que rotineira. 
Portando, o uso de escalas qualificadas para o rastreamento da violência contra os idosos como a Hawlek-Sengstock Elder Abuse Screening Test (H-S/EAST) devem fazer parte dos protocolos de assistência hospitalar a essa população. Podendo dessa forma permitir ações preventivas, coordenadas com os demais profissionais, bem como o encaminhamento correto de cada situação com intervenções eficazes para cada caso, evitando assim formas de intervenções tardias ou traumáticas. Além disso, permite o cumprimento do dever legal da profissão, do papel cidadão e o cuidado centrado na necessidade do paciente idoso, identificando fatores de risco para o abuso direto, como a depressão e o estresse.

O hospital também tem responsabilidade no combate à violência contra a pessoa idosa, portanto, deve capacitar seus profissionais para o enfrentamento desse problema, respaldados na compreensão das relações sociais conflituosas, o que se faz urgente e necessário diante dos resultados apresentados nesta pesquisa.

\section{AGRADECIMENTOS}

Nossos agradecimentos ao Hospital São Paulo, São PauloSP, Brasil.

\section{FINANCIAMENTO}

Bolsa de Iniciação Científica concedida a Isabela Granado Antequera, Programa Institucional de Bolsas de Iniciação Científica (PIBIC), Universidade Federal de São Paulo, concedida pelo Conselho Nacional de Desenvolvimento Científico e Tecnológico (CNPq).

\section{CONTRIBUIÇÕES DOS AUTORES}

Desenho do estudo. Isabela Granado Antequera. Maria Carolina Barbosa Teixeira. Ruth Ester Assayag Batista. Cassia Regina Vancini Campanharo. Meiry Fernanda Pinto Okuno

Coleta ou produção dos dados. Isabela Granado Antequera

Análise de dados e interpretação dos resultados. Isabela Granado Antequera. Maria Carolina Barbosa Teixeira Lopes. Ruth Ester Assayag Batista. Cassia Regina Vancini Campanharo. Paula Cristina Pereira da Costa. Meiry Fernanda Pinto Okuno

Redação e revisão crítica do manuscrito. Isabela Granado Antequera. Maria Carolina Barbosa Teixeira Lopes. Ruth Ester Assayag Batista. Cassia Regina Vancini Campanharo. Paula Cristina Pereira da Costa. Meiry Fernanda Pinto Okuno

Aprovação da versão final do artigo. Isabela Granado Antequera. Maria Carolina Barbosa Teixeira Lopes. Ruth Ester Assayag Batista. Cassia Regina Vancini Campanharo. Paula Cristina Pereira da Costa. Meiry Fernanda Pinto Okuno

Responsabilidade por todos os aspectos do conteúdo e a integridade do artigo publicado. Isabela Granado Antequera. Maria Carolina Barbosa Teixeira Lopes. Ruth Ester Assayag Batista. Cassia Regina Vancini Campanharo. Paula Cristina Pereira da Costa. Meiry Fernanda Pinto Okuno

\section{EDITOR ASSOCIADO}

\author{
Gerson Luiz Marinho
}

\section{REFERÊNCIAS}

1. Duarte MGM, Gouveia MAC, Andrade SAL. Population aging in Brazil current and future social challenges and consequences. Rev Bras Geriatr Gerontol. 2016 jun;19(3):507-19.

2. Castro VC, Rissardo LK, Carreira L. Violence against the Brazilian elderlies: an analysis of hospitalizations. Rev Bras Enferm. 2018;71(2, Supl. 2):777-85. http://dx.doi.org/10.1590/0034-7167-2017-0139. PMid:29791630.

3. Ministério da Mulher, da Família e dos Direitos Humanos (BR). Balanço Geral de Denúncias de violações contra a pessoa idosa no período de 2011 a 2018 [Internet]. Brasília: MDH; 2019 [citado 2020 mar 15] Disponível em: https://www.gov.br/mdh/pt-br/assuntos/noticias/2019/ junho/balanco-anual-do-disque-100-registra-aumento-de-13-emdenuncias-de-violacoes-contra-a-pessoa-idosa

4. Santos RC, Menezes RM, Araújo GK, Marcolino EC, Xavier EG, Gonçalves $R G$ et al. Síndrome da fragilidade e fatores associados em idosos no pronto atendimento. Acta Paul Enferm. 2020;33:eAPE20190159. http:// dx.doi.org/10.37689/acta-ape/2020AO0159.

5. Lei no 12.461 de 2011 (BR). Dispõe sobre a notificação compulsória dos atos de violência contra o idoso. Diário Oficial da União [periódico na internet], Brasília (DF), 26 jul 2011 [citado 2020 abr 17]. Disponíve em: http://www.planalto.gov.br/ccivil_03/_Ato2011-2014/2011/Lei/ L12461.htm

6. Diel M, Barbiani R. Violência familiar contra a pessoa idosa: expressões do fenômeno e perspectivas para o seu enfrentamento. Textos \& Contextos. 2018 dez;17(2):379-92. http://dx.doi.org/10.15448/16779509.2018.2.27484.

7. Silva RM, Oliveira DWD, Biscaro PCB, Ori NP, Pinto ANS, Jorge MLR Epidemiological survey in elderly people (II): oral health, anxiety, depression, stress, and drug utilization. Sci Med. 2016 mar;26(1):21980. http://dx.doi.org/10.15448/1980-6108.2016.1.21980.

8. Terassi M, Rossetti ES, Luchesi BM, Gramani-Say K, Hortense P, Pavarin $\mathrm{SCl}$. Factors associated with depressive symptoms in elderly caregivers with chronic pain. Rev Bras Enferm. 2020 jan;73(1):e20170782. http:// dx.doi.org/10.1590/0034-7167-2017-0782. PMid:31994674.

9. Ramos FP, Silva SC, Freitas DF, Gangussu LMB, Bicalho AH, Sousa $\mathrm{BVO}$ et al. Factors associated with depression in the elderly. REAS. 2019 jan;19(19):e239. http://dx.doi.org/10.25248/reas.e239.2019.

10. Billett MC, Campanharo CRV, Lopes MCBT, Batista REA, Belasco AGD, Okuno MFP. Functional capacity and quality of life of hospitalizes octogenarians. Rev Bras Enferm. 2019 dez;72(Supl. 2):48-54. http:// dx.doi.org/10.1590/0034-7167-2017-0781. PMid:31826190.

11. Carmona-Torres JM, Carvalhal-Silva RM, Vieira-Mendes MH, RecioAndrade B, Goergen T, Rodríguez-Borrego MA. Elder abuse within the family environment in the Azores Islands. Rev Latino-Am. Enfermagem. 2017 jan;25(0):e2932. http://dx.doi.org/10.1590/1518-8345.1871.2932.

12. Yon $\mathrm{Y}$, Mikton $\mathrm{CR}$, Gassoumis ZD, Wilber KH. Elder abuse prevalence in community settings: a systematic review and meta-analysis. Lancet Glob Health. 2017;5(2):e147-56. http://dx.doi.org/10.1016/S2214109X(17)30006-2. PMid:28104184.

13. Santos RM, Adamczyk SP, Silva FB, Lopes JCM, Nascimento KF. Nursing work of silente seffering in the elderly. Rev Gestão \& Saúde [Internet]. 2019; [citado 2020 mar 15];20(2):[aprox.9 telas]. Disponível em: http://www.herrero. com.br/files/revista/file85deb3138296b6e159edd5df6bb125a1.pdf

14. Reichenheim ME, Paixão Jr JCM, Moraes CL. Adaptação transcultural para o português (Brasil) do instrumento Hwalek-Sengstock Elder Abuse Screening Test (H-S/EAST) utilizado para identificar risco de violência contra o idoso. Cad Saude Publica. 2008 ago;24(8):1801-13. http:// dx.doi.org/10.1590/S0102-311X2008000800009. PMid:18709221.

15. Luft CD, Sanches SO, Mazo GZ, Andrade A. Versão brasileira da Escala de Estresse Percebido: tradução e validação para idosos. Rev Saude Publica. 2007 ago;41(4):606-15. http://dx.doi.org/10.1590/ S0034-89102007000400015. PMid:17589759. 
16. Paradela EMP, Lourenço RA, Veras RP. Validation of geriatric depression scale in a general outpatient clinic. Rev Saude Publica. 2005 dez;39(6):918-23. http://dx.doi.org/10.1590/S0034-89102005000600008. PMid:16341401.

17. Teixeira DB, Cruz SPL. Atenção à saúde do homem: análise da sua resistência na procura dos serviços de saúde. Rev Cubana Enferm [Internet]. 2016; [citado 2020 jul 27];32(4). Disponível em: http://www. revenfermeria.sld.cu/index.php/enf/article/view/985

18. Silva GCN, Almeida VL, Brito TRP, Godinho MLSC, Nogueira DA, Chini LT. Violência contra idosos: uma análise documental. Aquichan. 2018 dez;18(4):449-60. http://dx.doi.org/10.5294/aqui.2018.18.4.7.

19. Francisco PMSB, Segri NJ, Borim FSA, Malta DC. Prevalência simultânea de hipertensão e diabetes em idosos brasileiros: desigualdades individuais e contextuais. Cien Saude Colet. 2018 nov;23(11):3829-40. http://dx.doi.org/10.1590/1413-812320182311.29662016.

20. Guimarães DBO, Mendes PN, Rodrigues IS, Feitosa CDA, Sales JCS, Figueiredo MLF. Caracterização da pessoa idosa vítima de violência. Rev Enfermagem UFPE. 2016 abr;10(Supl. 3):1343-50. http://dx.doi. org/10.5205/reuol.7057-60979-3-SM-1.1003sup201601.

21. Faria PM, Dias FA, Molina NPFM, Nascimento JS, Tavares DMS. Quality of life and frailty among hospitalizes elderly patients. Rev Eletr Enf. 2016 dez;18:e1195. http://dx.doi.org/10.5216/ree.v18.38214.

22. Rodrigues RAP, Monteiro EA, Santos AMR, Pontes MLF, Fhon JRS, Bolina AF et al. Older adults abuse in three Brazilian cities. Rev Bras
Enferm. 2017 ago;70(4):783-91. http://dx.doi.org/10.1590/0034-71672017-0114. PMid:28793109.

23. Campos AC, Ferreira EF, Vargas AM, Gonçalves LH. Perfil do envelhecimento saudável de idosos brasileiros octagenários. Rev Lat Am Enfermagem. 2016 ago;24:e2724. http://dx.doi.org/10.1590/15188345.0694.2724. PMid:27579929.

24. Silva AR, Sampaio LS, Reis LA, Sampaio TSO. Violência contra idosos: associação entre o gênero dos agressores e o tipo de violência. Rev Multi Psic. 2017 nov;11(38):701-12. http://dx.doi.org/10.14295/idonline. v11i38.967.

25. Rosa PH, Beuter M, Benetti ERR, Bruinsma JL, Venturini L, Backes C. Stressors factors experience by hospitalizes elderly from the perspective of the Neuman Systems Model. Esc Anna Nery. 2018 nov;22(4):e20180148. http://dx.doi.org/10.1590/2177-9465-ean-2018-0148.

26. Teixeira JJM, Bastos GCFC, Souza ANL. Perfil de internação de idosos. Rev Soc Bras Clin Med [Internet]. 2017 Mar; [citado 2020 abr 18];15(1):[aprox. 5 telas]. Disponível em: http://www.sbcm.org.br/ojs3/ index.php/rsbcm/article/view/245

27. Serra MA, Nascimento IMT, Guimarães GL, Silva SM, Goveia VR, Mendoza IYQ. Prevalence of depressive symptoms in hospitalized older adults: a comparative study. Rev Enferm UERJ. 2019 abr;27:e36091. http://dx.doi.org/10.12957/reuerj.2019.36091. 